\title{
DEMOCRACY IN CHINA
}





\section{DEMOCRACY IN CHINA \\ The Coming Crisis}

JIWEI CI 
Copyright $\odot 2019$ by the President and Fellows of Harvard College All rights reserved

Printed in the United States of America

First printing

Jacket design: Graciela Galup

Jacket art: Jon Paget/Getty Images

9780674242159 (EPUB)

9780674242166 (MOBI)

$9780674242142(\mathrm{PDF})$

$\dot{\alpha}$ e Library of Congress has cataloged the printed edition as follows:

Names: Ci, Jiwei, 1955- author.

Title: Democracy in China : the coming crisis / Jiwei Ci.

Description: Cambridge, Massachusetts : Harvard University Press, 2020. |

Includes bibliographical references and index.

Identifiers: LCCN 2019014446 | ISBN 9780674238183 (alk. paper)

Subjects: LCSH: Democracy-China-History-21st century. | DemocratizationChina-History-21st century. | Legitimacy of governments-China-History21st century. | Zhongguo gong chan dang.

Classification: LCC JC423 .C56736 2020 | DDC 320.951-dc23 LC record available at https://lccn.loc.gov/2019014446 